\title{
Human Body Heat Detection by using Thermal Image in Crashed Building
}

\author{
Sagar Shankarrao Dake
}

\author{
Hemanta Kumar \\ Mohanta
}

\author{
Sushma Yadav
}

\begin{abstract}
From the last few years, earthquakes are occur in many countries, up to $85 \%$ building are crashed and in that building lot of human are stuck, injured or may be dead. So in that way where these humans are stuck it's not possible to find in that building, in this paper thermal imaging are used to detect the human body heat, then they will easily detected and they will rescue from that building. In crashed building the position prediction are used to determine the human body position and after rescue face identification are used with supervised classification for shape factor.
\end{abstract}

\section{Keywords:}

Position prediction, Face Identification

\section{INTRODUCTION}

Earthquakes, also called temblors, can be so tremendously destructive; it's hard to imagine they occur by the thousands every day around the world, usually in the form of small tremors.

Some $80 \%$ of all the planet's earthquakes occur along the rim of the Pacific Ocean, called the "Ring of Fire" because of the preponderance of volcanic activity there as well. Most earthquakes occur at fault zones, where tectonic plates-giant rock slabs that make up the Earth's upper layer - collide or slide against each other. These impacts are usually gradual and unnoticeable on the surface; however, immense stress can build up between plates. When this stress is released quickly, it sends massive vibrations, called seismic waves, often hundreds of miles through the rock and up to the surface. Other quakes can occur far from faults zones when plates are stretched or squeezed. Scientists assign a magnitude rating to earthquakes based on the strength and duration of their seismic waves. A quake measuring 3 to 5 is considered minor or light; 5 to 7 is moderate to strong; 7 to 8 is major; and 8 or more is great. On average, a magnitude 8 quake strikes somewhere every year and some 10,000 people die in earthquakes annually. Collapsing buildings claim by far the majority of lives, but the destruction is often compounded by mud slides, fires, floods, or tsunamis. In this paper, after earthquake building crashed, if there any human is live in crashed building then, at that time thermal image will detect the body heat, then this person will rescue from that building. Smaller temblors that usually occur in the days following a large earthquake can complicate rescue efforts and cause further death and destruction. Loss of life can be avoided through emergency planning, education, and the construction of buildings that sway rather than break under the stress of an earthquake. Earthquakes are measured using observations from seismometers. The moment magnitude is the most common scale on which earthquakes larger than approximately 5 are reported for the entire globe. The more numerous earthquakes smaller than magnitude 5 reported by national seismological observatories are measured mostly on the local magnitude scale, also referred to as the Richter magnitude scale. These two scales are numerically similar over their range of validity. Magnitude 3 or lower earthquakes are mostly almost imperceptible or weak and magnitude 7 and over potentially causes serious damage over larger areas, depending on their depth. The largest earthquakes in historic times have been of magnitude slightly over 9 , although there is no limit to the possible magnitude. The most recent large earthquake of magnitude 9.0 or larger was a 9.0 magnitude earthquake in Japan in 2011 (as of March 2014), and it was the largest Japanese earthquake since records began. Intensity of shaking is measured on the modified Mercalli scale. The shallower an earthquake, the more damage to structures it causes, all else being equal. It is estimated that around 500,000 earthquakes occur each year, detectable with current instrumentation. About 100,000 of these can be felt. Minor earthquakes occur nearly constantly around the world in places like California and Alaska in the U.S., as well as in Salvador, Mexico, Guatemala, Chile, Peru, Indonesia, Iran, Pakistan, the Azores in Portugal, Turkey, New Zealand, Greece, Italy, India and Japan, but earthquakes can occur almost anywhere, including Downstate New York, England, and Australia. Larger earthquakes occur less frequently, the relationship being exponential; for example, roughly ten times as many earthquakes larger than magnitude 4 occur in a particular time period than earthquakes larger than magnitude 5. In the (low seismicity) United Kingdom, for example, it has been calculated that the average recurrences are: an earthquake of 3.7-4.6 every year, an earthquake of 4.7-5.5 every 10 years, and an earthquake of 5.6 or larger every 100 years.

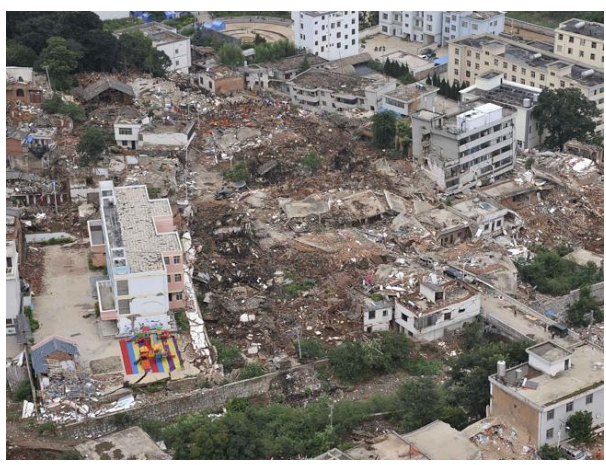

Figure1. After Earthquake

\section{POSITION PREDICTION [2]:}

The accurate prediction leads the tracking system to desire stability. The position prediction is based on gray theory, it assumes the parameter internal structure and characteristic of observed system that are unknown, or else it is called "black system". The sensory information can measure external performance of system and it finds a suitable model to approximate its dynamic. The approximate model is also called the "white system". The optimal parameter of 
white system is calculated from so-called "grey model". The grey model is written as GM $(\alpha, \beta)$ where, $\beta$ is number of variable equation and $\alpha$ is order. In the implementation stage $\beta$ variable differential equation and $\alpha$ is the modelling equation. The higher the assigned $\alpha$, the more sensitive to the input data will the obtained model become. The GM $(1,1)$ is widely used and in application its successfully demonstrate such as forecasting, earthquake prediction. The Time-varying scalar is used for tracking a moving target, from the sensory information should be $\mathrm{x}(\mathrm{t})$ where $(\mathrm{t}=1,2, \ldots . \mathrm{n})$ and the accumulated generating operation is define as accumulated measurement $z(k)=$ $\sum_{t=1}^{k}, x(t)$ for $(\mathrm{k}=1,2, \ldots \mathrm{n})$. We then model the dynamic change of $\mathrm{z}(\mathrm{t})$ by the following first-order ordinary differential equation

$$
\frac{d z(t)}{d t}+a z(t)=b
$$

In grey theory, (1) is called the "white descriptor" for modelling a white system and we can estimate parameters $a$ and $b$ from the observed system outputs. However, to estimate the parameters of the unknown system only from the sensory measurements it is approximated by the following grey-differential equation.

$$
\begin{array}{ll}
\frac{d z(t)}{d t}+a g(t)=b & \text { where } g(t)=(z(t+1)+ \\
z(t)) / 2 & \text { (2) }
\end{array}
$$

To estimate the optimal parameters $a$ and $b$, the minimum least-square method can be applied by introducing the accumulated generating operation in a time interval. For simplification purposes, the sampled time of past measurement $X$ is taken as a unit. The first term of (2) in a discrete system can be written as

$$
\frac{d z(t)}{d t}=z(t+1)-z(t)=x(t+1)(3)
$$

Equation (2) can be rewritten as

$$
\mathrm{x}(\mathrm{t}+1)=\mathrm{a}[-1 / 2(\mathrm{z}(\mathrm{t}+1)+\mathrm{z}(\mathrm{t}))]+\mathrm{b}
$$

Substituting the sequential data $\mathrm{X}$ and $\mathrm{Z}$ into (4), we get the following matrix relation

$$
\left[\begin{array}{c}
x(2) \\
x(3) \\
\cdot \\
\cdot \\
\cdot \\
x(n)
\end{array}\right]=\left[\begin{array}{cc}
-\frac{1}{2}(z(2)+z(1)) & 1 \\
-\frac{1}{2}(z(3)+z(2)) & 1 \\
\cdot & \\
\cdot & \\
-\frac{1}{2}(z(n)+z(n-1)) & 1
\end{array}\right]\left[\begin{array}{l}
a \\
b
\end{array}\right](5)
$$

Equation (5) can be rewritten as

$$
Y=[x(2) \quad x(3) \ldots x(n)]^{T}=B \emptyset
$$

Where B is as shown at the bottom of the page and

$$
\varnothing=\left[\begin{array}{ll}
a & b
\end{array}\right]^{T}
$$

For the case of $\mathrm{n} \geq 2$, we can apply ordinary least-square estimation with a linear model $\mathrm{Y}=\mathrm{B} \emptyset$, where the sum of squares function is defined by the following quadratic equation

$$
\varsigma L S=Y-B \phi)^{T}(Y-B \phi)
$$

The optimal solution can be obtained by minimizing the $\varsigma L S$ using the matrix derivation $\nabla$ with respect to $\phi$ as the following equations:

$$
\begin{gathered}
\nabla \emptyset_{\varsigma} L S=2\left[\nabla_{\emptyset}(Y-B \emptyset)^{T}\right\rfloor[Y-B \emptyset] \\
\nabla_{\varnothing}(Y-B \emptyset)^{T}=-\nabla_{\varnothing} \emptyset^{T} B^{T}=-B^{T}
\end{gathered}
$$

Setting (8) to zero, the optimal parameters of of $\phi$ the grey model can be obtained by the following equation

$$
\widehat{\emptyset}=\left[\begin{array}{ll}
\hat{a} & \hat{b}
\end{array}\right]^{T}=\left(B^{T} B\right)^{-1} B^{T} Y
$$

The estimated parameters are then brought into the response solution of the first-order ordinary differential equation (1) for prediction of the accumulated generating operation

$$
\hat{z}(n+1)=\left(z(1)-\frac{\hat{b}}{\hat{a}}\right) e^{-\hat{a} n}+\frac{\hat{b}}{\hat{a}}(11)
$$

\section{IMAGE ACQUISITION AND ANALYSIS SYSTEM [1]:}

In this paper, the thermal images of human face were produced by thermal video system with IR. The thermal image principle is comes from well-known law by Stefan and Boltzmann, it is represented by equation,

$$
W=\epsilon \cdot \sigma \cdot T^{4}
$$

Where, $\mathrm{W}$ is radiant emittance $\left(\mathrm{W} / \mathrm{cm}^{2}\right), \epsilon$ is emissivity, $\mathrm{T}$ is temperature (k), $\sigma$ is Stefan-Boltzmann constant $\left(=5.6705 \times 10^{-12} \mathrm{~W} / \mathrm{cm}^{2} \mathrm{k}^{4}\right) . \epsilon$ is estimated for human skin at 0.98 to 0.99 . In this paper, for human skin approximate value of 1 was used as $\epsilon$. The values of $\epsilon$ for almost all substances except human skin temperature is select for producing the thermal image at that time human face is easily extracted. Using the value of 1 for $\epsilon$. Faceimage were observed by a monitor 


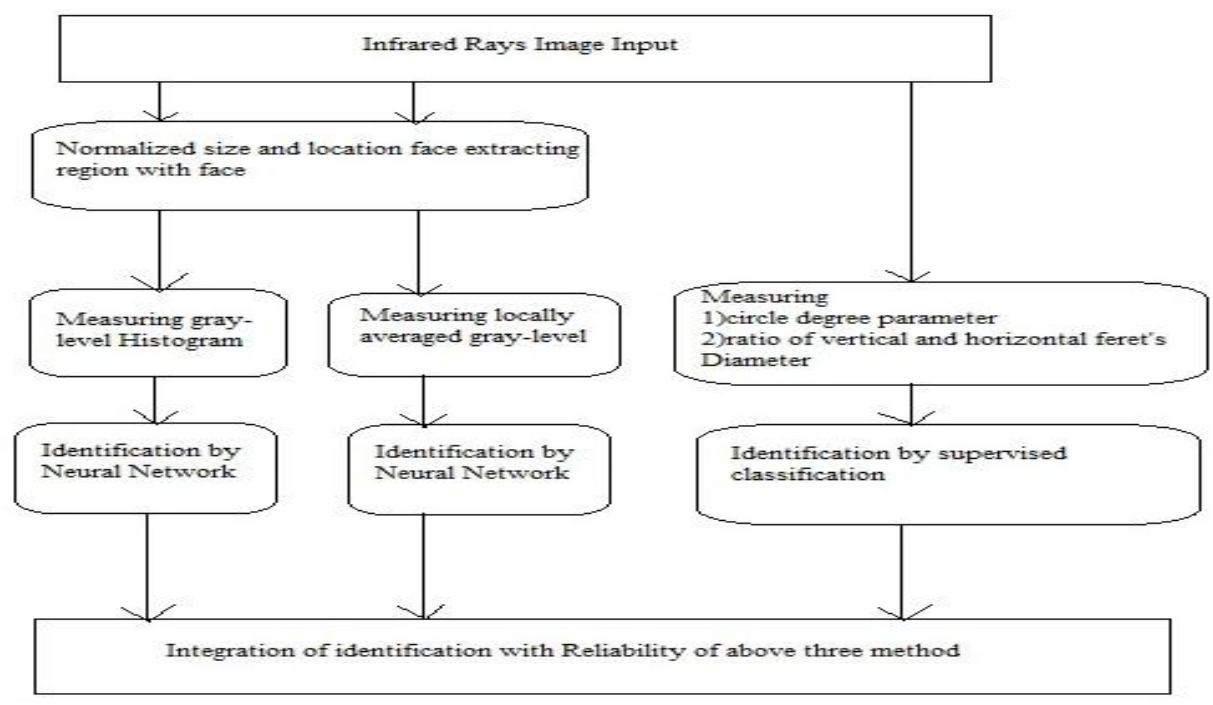

Figure 2. Flowchart of Face Identification

through an IR thermal image system with 16 (4 bits) as thermal level and recorded $8 \mathrm{~mm}$ video and then digitized with 256 level ( 8 bits) per pixel. Then this digital image is stored on fixed spatial resolution of 300x300 pixel element and

then processed by a personal computer. The input images were produced under the operating condition which presented lower- gray-level for colder part and higher-gray level for hotter part. The temperature IR is does not depend on skin colour, darkness and lighting condition in the input image conditioning in the resulting in that face and its surrounding.

\section{RECOGNITION ALGORITHM}

Figure 2 shows the flowchart, for face identification. The face is finally identified through integrating information from $\mathrm{NN}$ for gray-level histogram of face, $\mathrm{NN}$ for mosaic image of face and supervised classification for shape factors of face.

\subsection{Face Identification with Supervised Classification for Shape Factor}

The input image of the face is segmented with Otsu's method, followed by measuring SF1. The circle degree parameter calculated with area and perimeter, and SF2. The ratio of vertical and horizontal Feret's diameters. SF1 and SF2 are calculated as follow

$$
\begin{aligned}
& S F 1=\frac{4 \pi A}{P^{2}} \\
& S F 2=\frac{F H}{F V}
\end{aligned}
$$

Where A is area, $\mathrm{P}$ is perimeter, $F H$ is horizontal Feret's diameter and $F V$ is vertical Feret,'s diameter. Then, Euclidean distance $D_{i}$ defined as below is used as discriminant, function for face identification.

$$
D_{i}=\sqrt{\left(\frac{S F 1_{i}}{S \bar{F} 1}-\frac{S F 1 M}{S \bar{F} 1}\right)^{2}+\left(\frac{S \bar{F} 2_{i}}{S \bar{F} 2}-\frac{S F 2 M}{S \bar{F} 2}\right)^{2}}
$$

where $S \bar{F} 1$ is tlie average value of SF1 of learning data, $S \bar{F} 2$ is average value of SF2 of learning data, $S F 1_{i}$ is the average value of SF1 of learning data belonging to person i, $S \bar{F} 2$ is the average value of SF2 of learning data belonging to person i, $S F 1 M$ is the measured value of $S F 1$ for unknown person and $S F 2 M$ is the measured value of SF2 for unknown person. Using Euclidean distance as discriminant, function where the element of characteristic vector is normalized by the corresponding average value of learning data, the face is identified with supervised classification through comparing the test, data with the averaged data of each learned class. The unknown person is judged as person $\mathrm{x}$ when $D_{x}$, presents the smallest value among Di.

\section{CONCLUSION}

The proposed work is described theoretically, to secure human from the crashed building by using thermal image. The position prediction is used to determine the human body position and face identification is used to identify the human identity.

\section{REFERENCES}

[1] Y. Yoshitomi, T. Miyaura, S. Tomita, S. Kimura, "Face Identification Using Thermal Image Processing".

[2] Ren C. Luo, Fellow, IEEE, and Tse Min Chen, "Autonomous Mobile Target Tracking System Based on Grey-Fuzzy Control Algorithm". 